\title{
Fluorescence Based on Surface Plasmon Coupled Emission for Ultrahigh Sensitivity Immunoassay of Cardiac Troponin I
}

\author{
Vien Thi Tran ${ }^{1,2}$ and Heongkyu Ju ${ }^{1,2, *(D)}$ \\ 1 Department of Physics, Gachon University, Seongnam-si, Gyeonggi-do 13120, Korea; tranvien04@gmail.com \\ 2 Gachon Bionano Research Institute, Gachon University, Seongnam-si, Gyeonggi-do 13120, Korea \\ * Correspondence: batu@gachon.ac.kr; Tel.: +82-31-750-8552
}

Citation: Tran, V.T.; Ju, H.

Fluorescence Based on Surface Plasmon Coupled Emission for Ultrahigh Sensitivity Immunoassay of Cardiac Troponin I. Biomedicines 2021, 9, 448. https://doi.org/10.3390/ biomedicines 9050448

Academic Editor: Sharon Marx

Received: 18 March 2021

Accepted: 19 April 2021

Published: 21 April 2021

Publisher's Note: MDPI stays neutral with regard to jurisdictional claims in published maps and institutional affiliations.

Copyright: (c) 2021 by the authors. Licensee MDPI, Basel, Switzerland. This article is an open access article distributed under the terms and conditions of the Creative Commons Attribution (CC BY) license (https:/ / creativecommons.org/licenses/by/ $4.0 /)$.

\begin{abstract}
This work demonstrates the quantitative assay of cardiac Troponin I (cTnI), one of the key biomarkers for acute cardiovascular diseases (the leading cause of death worldwide) using the fluorescence-based sandwich immune reaction. Surface plasmon coupled emission (SPCE) produced by non-radiative coupling of dye molecules with surface plasmons being excitable via the reverse Kretschmann format is exploited for fluorescence-based sandwich immunoassay for quantitative detection of cTnI. The SPCE fluorescence chip utilizes the gold ( $2 \mathrm{~nm})$-silver $(50 \mathrm{~nm})$ bimetallic thin film, with which molecules of the dye Alexa 488 (conjugated with detection antibodies) make a near field coupling with the plasmonic film for SPCE. The experimental results find that the SPCE greatly improves the sensitivity via enhancing the fluorescence signal (up to 50-fold) while suppressing the photo-bleaching, permitting markedly enhanced signal-to-noise ratio. The limit of detection of $21.2 \mathrm{ag} \mathrm{mL}^{-1}$ (atto-gram mL ${ }^{-1}$ ) is obtained, the lowest ever reported to date amid those achieved by optical technologies such as luminescence and label-free optical sensing techniques. The features discovered such as ultrahigh sensitivity may prompt the presented technologies to be applied for early diagnosis of $\mathrm{cTnI}$ in blood, particularly for emergency medical centers overloaded with patients with acute myocardial infarction who would suffer from time-delayed diagnosis due to insufficient assay device sensitivity.
\end{abstract}

Keywords: fluorescence; cardiac biomarker; Troponin I; surface plasmon coupled emission; lab-onchip optical biosensor

\section{Introduction}

Cardiovascular diseases, including acute coronary syndrome, have been considered the globally leading cause of human death and have been gathering great attention for their early diagnosis with cardiac markers such as myoglobin [1-3], C-reactive protein [4,5], B-type natriuretic peptide [6,7], cardiac Troponin T [8,9], and Troponin I (cTnI) [10,11]. Amid the biomarkers, cTnI has been conceived as one of the most important biomarkers for early diagnosing acute myocardial infarction (and stroke) due to its response sensitivity and specificity [12,13]. The cTnI concentration that remains at $1 \mathrm{pg} \mathrm{mL}^{-1}$ in serum of a normal person basically grows in response to damage to cardiac muscle and can be measured using the currently available test devices such as chemo-luminescence immunoassay and electrocardiogram.

Their limited sensitivity $\left(\sim 100 \mathrm{pg} \mathrm{mL}^{-1}\right)$ of the test devices and its detection time of a couple of hours [12], however, would not allow the cTnI level $\leq 10 \mathrm{pg} \mathrm{mL}^{-1}$ in serum to be reliably measured 1-2 $\mathrm{h}$ after onset of the symptom such as a heart attack (whereupon its level reaches $\sim 100 \mathrm{ng} \mathrm{mL}{ }^{-1} 3-4 \mathrm{~h}$ after the onset) [14]. Thus, the high sensitivity assay device and the related technologies have come into a key play for early diagnosis of urgent cases of cardiovascular diseases. Extensive efforts have been made in an attempt to enhance the sensitivity in detecting CTnI concentration using the assays including electrochemical method [15,16], transistor based assay [17,18], chemiluminescence [19], fluorescence 
immunoassay [20], colorimetric assay [21], surface plasmon resonance (SPR) [22,23], and optical microfiber based label-free assay [10], while point-of-care testing of cTnI was reported for test device portability without compromising the sensitivity [24].

The fluorescence technique that lab-on-chip assays would adopt as the primary workhorse is known to suffer from difficulties in efficient light collection and dye photobleaching. These challenges, however, may be resolved by surface plasmon coupled emission (SPCE) that produces the metal enhanced fluorescence due to dye-surface plasmon near field interaction. This nano-photonics technology has been explored to amplify the fluorescence signal via applying near field plasmonic technologies to the fluorescence assay without influencing analytes $[25,26]$. The excited dye molecules in close proximity to a thin metal film couples with it in a non-radiative manner, generating surface plasmons at emission wavelengths $\left(\lambda_{\mathrm{em}}\right)$. This near field coupling produces the directional emission at wavelengths identical to $\lambda_{\mathrm{em}}$, into an adjacent medium of a high refractive index through the metal film. The directional emission has the angles determined by the photon momentum conservation $[27,28]$. These angles would be identical to the incident angles of light for surface plasmon resonance (SPR) excited at $\lambda_{\mathrm{em}}$ in a Kretschmann configuration, reflecting the SPCE as the time-reversal operation of the SPR [29,30].

The SPCE that occurs via the non-radiative decay channel of excited dye molecules would increase both the fluorescence power detected and the light collection efficiency owing to the feature of directional emission. It has also been unfolded that the SPCE would reduce the photo-bleaching effects into the enhanced dye stability.

In this paper, we present the fluorescence-based sandwich immunoassay of cTnI with the SPCE technique employed for signal amplification of 50-fold at maximum. The SPCE chip fabricated includes the cTnI-specific capture antibodies on the bimetallic thin films of $2 \mathrm{~nm}$-thick gold $(\mathrm{Au}) / 50 \mathrm{~nm}$-thick silver $(\mathrm{Ag})$ layers on a glass substrate. The bimetallic SPCE based sandwich immunoassay that greatly suppresses the photo-bleaching demonstrated the ultrahigh sensitivity, with the limit of detection (LOD) of $\sim 21.2$ attogram (ag) $\mathrm{mL}^{-1}$ via the SPCE-enhanced fluorescence at the wavelength centered at $522 \mathrm{~nm}$. This detection limit is lowest ever achieved to date amid those relying on optical technologies such as luminescence and label-free optical biosensor techniques. We also discuss how SPCE enhances the fluorescence signal from the sandwich assay that imposes the geometrical characteristics of molecules on the metal surface, such as the dye molecule-metal surface spacing.

The technologies presented also remove the need of using a dichroic beam splitter commonly used in a typical lab-on-chip fluorescence assay to separate emitted fluorescence from excitation source. Its simpler form potentially better suits a mini-sized assay device. The ultra-high sensitivity achievable with the SPCE chip can thus find a suitable application in biomedical assays using a simpler format of lab-on-chip based fluorescence technologies. In particular, the technologies presented for ultrahigh sensitivity assay of cTnI can open up possibility of errorless early diagnosis of $\mathrm{cTnI}$ in blood for effectively managing an emergency medical center, with its entry frequently overloaded with a large number of patients with acute cardiovascular diseases due to insufficient sensitivity of the current diagnostic devices.

\section{Materials and Methods}

\subsection{Materials and Reagents}

We purchased the two types of cTnI antibodies used for the sandwich immunoassay, i.e., capture antibody (clone 560, mouse) and detection antibody conjugated with the dye (Alexa fluor-488, clone 19C7, mouse) from Novus Co., (Centennial, CO, USA). Recombination Human cTnI was bought from Abcam Co., (Cambridge, UK). Cysteamine hydro-chloride $\left(\mathrm{C}_{2} \mathrm{H}_{7} \mathrm{NS} . \mathrm{HCl}, 98 \%\right)$, N-hydroxysuccinimide (NHS, $\left.\mathrm{C}_{4} \mathrm{H}_{5} \mathrm{NO}_{3}, 98 \%\right), \mathrm{N}$ (3-dimethylaminoproyl)- $\mathrm{N}^{\prime}$-ethylcarbodiimide hydrochloride (EDC, $\mathrm{C}_{8} \mathrm{H}_{17} \mathrm{~N}_{3} \cdot \mathrm{HCl}$, crystalline), (3-amininpropyl)triethoxysilance (APTES, 99\%), and propanol $\left(\mathrm{C}_{3} \mathrm{H}_{8} \mathrm{O}, 99 \%\right.$ ) were purchased from Sigma-Aldrich Co., (St. Louis, MO, USA). Phosphate buffered saline 
(PBS, 1X), ultra-pure filtered water, albumin (BSA, 98\%) were bought from Biosesang Co. (Seongnam-si, Korea). Pellets of Au (99.99\%), Ag (99.99\%) and Cr (99.99\%) used for plasmonic metal coating via thermal evaporation were purchased from Taewon Scientific Co., Ltd. (iTASCO, Seoul, Korea). Polydimethylsiloxane (PDMS) was purchased from Dow Corning Co., (Midland, MI, USA).

\subsection{Plasmonic Chip Fabrication}

We made the PDMS structure, which has a thick-walled hollow inner space with its open top looking like an equilateral hexagon (side length of $1 \mathrm{~cm}$ ) as shown in Figure 1a (top view). We used an oxygen plasma to bond it to a soda lime glass substrate (Marienfield, Germany), generating a chamber of a hexagonal pillar shape with its volume of $200 \mu \mathrm{L}$. A thermal evaporator (DAEKI HI-TECH Co., Ltd., Daejeon, Korea) was used to coat the film layers of $2 \mathrm{~nm}$-thick chromium (Cr), $50 \mathrm{~nm}$-thick Ag and $2 \mathrm{~nm}$-thick Au consecutively on glass bottom of the PDMS walled chamber at the deposition speed of $0.5 \AA \mathrm{s}^{-1}$ for both $\mathrm{Ag}$ and $\mathrm{Au}$, and $0.1 \AA^{-1} \mathrm{~s}^{-1}$ for $\mathrm{Cr}$ (monitored by a quartz crystal microbalance (QCM, QI8010, Fil-Tech Inc., Boston, MA, USA)), at the pressure $\leq 7 \times 10^{-6}$ Torr.

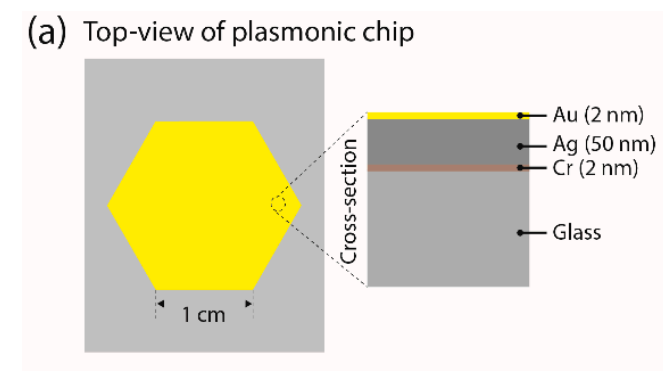

(c) Fluorescence image
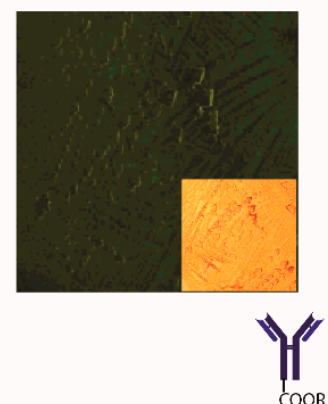

\section{(d) Fluorescence spectrum}

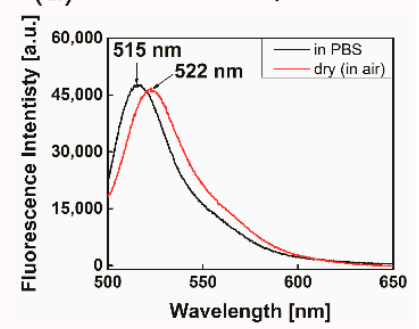

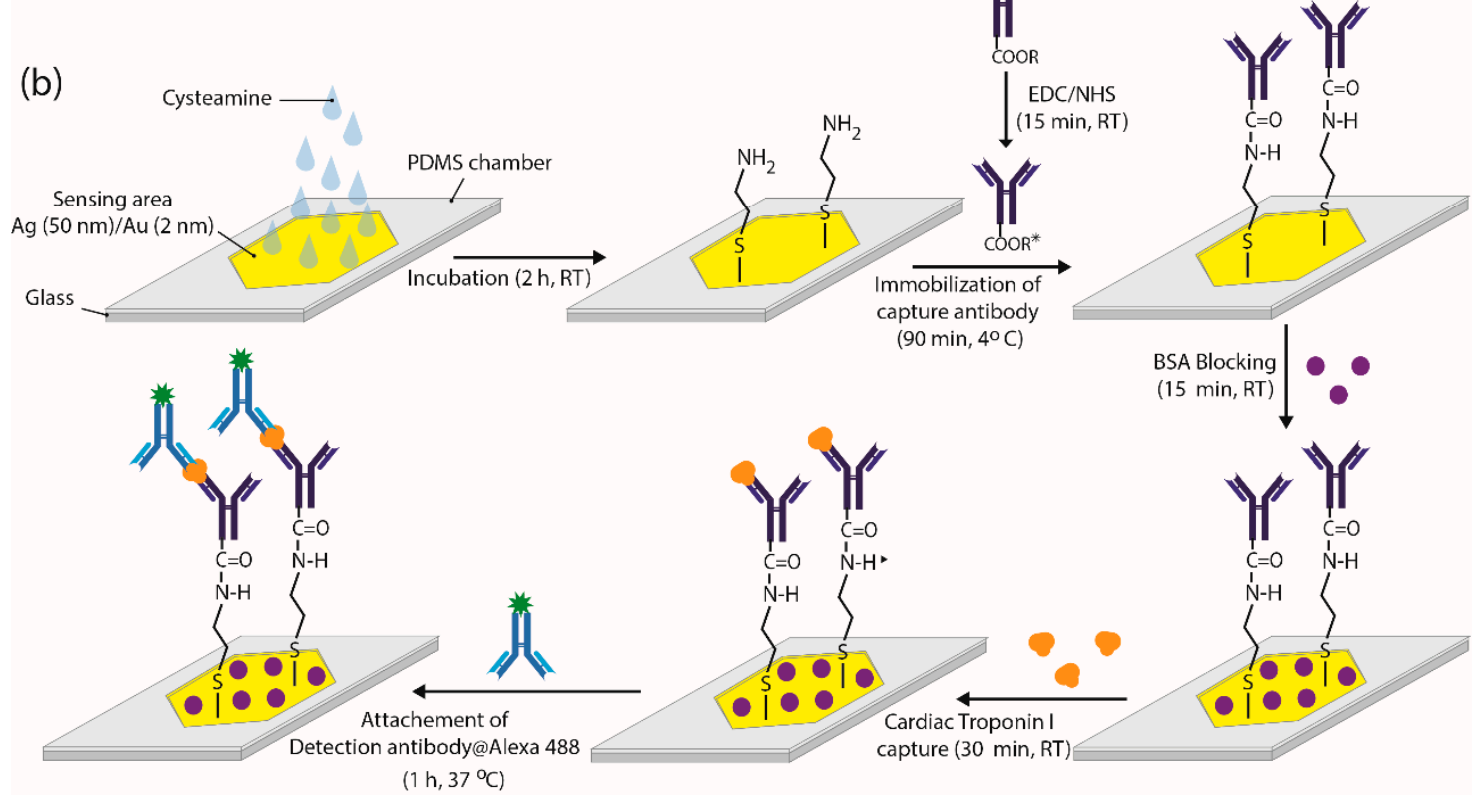

Figure 1. (a) Top-view and cross-section of the SPCE chip. (b) Procedures for the sandwich immune reaction for detecting cTnI. (c) Fluorescence microscope image of the green color due to fluorescence from the SPCE chip that went through the procedures of Figure 1b, under excitation (blue) light illumination (inset shows the image under white light illumination). (d) The fluorescence spectrum of Alexa 488 conjugated D-Ab of $1 \mu \mathrm{g} \mathrm{mL}^{-1}$ in PBS solution and in air under illumination of LED at $470 \mathrm{~nm}$ wavelength.

The $\mathrm{Cr}$ layer acts as an adhesive layer for coating the Ag layer on a glass substrate [31]. The $2 \mathrm{~nm}$-thick Au layer enables the thiol-linking to be used for immobilizing the capture probes, while acting as anti-oxidization protective layer for the inner Ag layer [26,32]. 
Use of the Ag layer as the prime metallic film for surface plasmon is supported by its higher quality factor, indicating a greater number of dye molecules coupled for more highly effective SPCE than in a case of an Au layer at visible wavelengths [26,32-36].

Care has to be taken to ensure that the illumination area by the dye excitation light source did not exceed the hexagonal area of the chamber bottom to avoid unwanted scattering of light with chamber wall.

\subsection{Procedures for the Sandwich Immune Reaction on the Surface of SPCE Chip}

Figure $1 \mathrm{~b}$ shows the procedures of the sandwich immunoassay of cTnI on the SPCE chip. Its outermost surface of Au film is modified using cysteamine of $10 \mathrm{mM}$ (diluted in deionized water) for $2 \mathrm{~h}$ at room temperature (RT), to generate amine group on it. The cTnI capture antibody $(\mathrm{C}-\mathrm{Ab})$ of $2 \mu \mathrm{g} \mathrm{mL} \mathrm{m}^{-1}$ concentration is introduced to the amine-modified surface. The $\mathrm{C}$ - $\mathrm{Ab}$ binding amine group is taken in $90 \mathrm{~min}$ at $4{ }^{\circ} \mathrm{C}$. Prior to immobilizing the $\mathrm{C}-\mathrm{Ab}$, we activate its carboxylic groups in $15 \mathrm{~min}$ by immersing it in the solution of 1,4-dioxane, toluene, 1-ethyl-3-(3-(dimethylamino)propyl) carbodiimide (EDC) and $\mathrm{N}$ hydroxysuccinimide (NHS) with phosphate buffered saline (PBS) solvent. The bovine serum albumin (BSA) of $2 \mathrm{mg} \mathrm{mL}^{-1}$ is in turn injected to the surface and incubated in $15 \mathrm{~min}$ as the blocking agents to avoid non-specific binding of cTnI with the Au surface. Then the target molecules and $\mathrm{CTnI}$ of various concentrations are injected to the $\mathrm{C}-\mathrm{Ab}$ modified surface in $30 \mathrm{~min}$. Next, the D-Ab@Alexa 488 of $1 \mu \mathrm{g} \mathrm{mL} \mathrm{m}^{-1}$ is injected in $1 \mathrm{~h}$ at $37^{\circ} \mathrm{C}$ to complete the sandwich immune reaction, with the chip surface being ready for the SPCE-enhanced fluorescence.

It is noted that the concentrations of the C-Ab and D-Ab@Alexa 488 all need to exceed that of $\mathrm{cTnI}$ injected to avoid unwanted saturation of cTnI assayed. All bio-agents are diluted in PBS solvent. The surface is rinsed with PBS solvent immediately after every immobilization procedure mentioned above. Finally, the SPCE chip is dried in an oven at $37^{\circ} \mathrm{C}$ for 30 min to make an air ambient that determines the SPCE directional angle, before being installed in the optical setup for fluorescence detection.

We also prepare the control chip to detect the fluorescence without SPCE enhancement, reflecting the fluorescence signal similar to that obtainable by a conventional fluorescence technique. Its signal magnitude is used as a reference to estimate the amplification factor of the SPCE-enhanced fluorescence. The control chip is designed to have the same layers as the SPCE chip, i.e., C-Ab with blocking agent, cTnI, and D-Ab@Alexa 488, except those of the bimetallic film. Therefore, for control chip fabrication, the slide glass surface undergoes the oxygen plasma treatment prior to being incubated in the APTES (volume concentration of $2 \%$ in propanol) to generate the amine-modified surface. We use the procedures for immobilizing, on the control chip, C-Ab, BSA (blocking agents), cTnI, and D-Ab@Alexa 488 , exactly in the same ways as those mentioned above for the SPCE chip.

As shown in Figure 1c, we make a visual check of the fluorescence from the dye on surface of the SPCE chip (that undergoes the procedures in Figure 1b), using a fluorescence microscope (Eclipse 80i, Nikon, Japan) designed to detect the fluorescence emitting into air ( $\mathrm{cTnI}$ concentration of $6.6 \mu \mathrm{g} \mathrm{mL}^{-1}$ ). Under illumination of blue excitation light, green fluorescence is clearly visible while its inset shows an image under white light illumination. Observation of the green fluorescence verifies the successful immobilization of D-Ab@Alexa 488 through the sandwich immune reaction described above.

Figure $1 \mathrm{~d}$ shows the spectra of fluorescence from D-Ab@Alexa 488 in dried condition, measured by a spectrometer (Ocean Optics USB 2000+, Orlando, FL, USA). The spectrum peaks at $515 \mathrm{~nm}$ when the D-Ab@Alexa 488 is in the PBS solvent. The peak shifts to $522 \mathrm{~nm}$ in dried condition [37]. Such a shift is taken into account to choose the pass band filter that allows only fluorescence light at $\lambda_{\mathrm{em}}$ while blocking light at $\lambda_{\mathrm{ex}}$ for detection. It is worth noting that the extremely low levels of the cTnI concentrations ranging from $0.01 \mathrm{pg} \mathrm{mL}^{-1}$ to $0.5 \mathrm{pg} \mathrm{mL}^{-1}$ (PBS buffer) cannot serve for the visual check of the dye green color when using the fluorescence microscope. It is noted that, for a given excitation power, at each concentration (including zero concentration), three independent measurements are 
conducted to obtain an error bar. Each measurement includes recording the signal for $10 \mathrm{~s}$ with the data sampling frequency of $1 \mathrm{~Hz}$.

\subsection{Instrument and Apparatus for Detecting SPCE-Enhanced Fluorescence}

Figure $2 \mathrm{a}, \mathrm{b}$ shows schematic and the photo of the setup for detecting the SPCEenhanced fluorescence. The fluorescence excitation source is the light emitting diode (LED) with its central wavelength of $470 \mathrm{~nm}$ (M470L3, Thorlabs Inc., Newton, MA, USA), its output power being controllable via a current supplier. The source light passes through the excitation filter (EXF) $(470 \mathrm{~nm} \pm 20 \mathrm{~nm}$, Chroma Co., Bellows Falls, VT, USA) into a circular cross-sectional beam of $1 \mathrm{~cm}$ diameter. The adjustable iris with which we follow the EXF could control the illumination area on surface of the SPCE chip. The chip is installed on one side surface of a right-angled prism of N-BK7. Index-matching oil (MOIL-10LF, Leica Type F, Thorlabs Inc., Newton, MA, USA) is inserted to fill the gap between the glass bottom of the chip and the prism surface to avoid unwanted total internal reflection induced by index discontinuity.
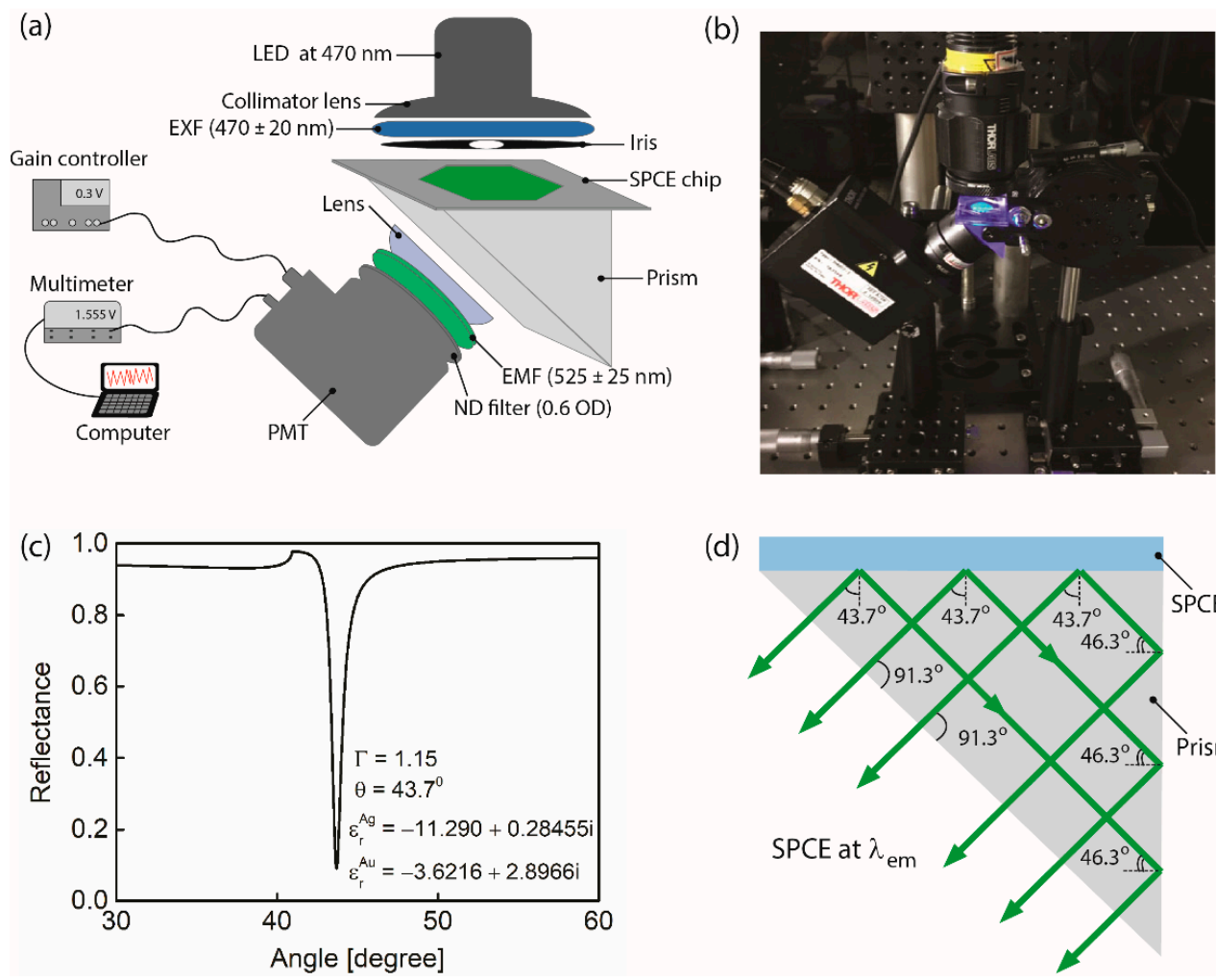

(d)

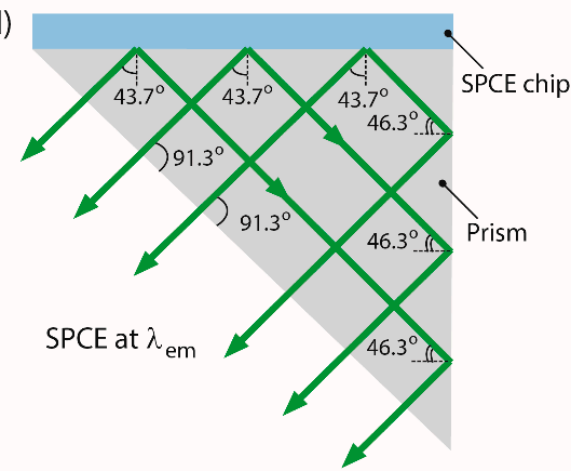

Figure 2. (a) Schematic of the experimental setup (ND filter: neutral density filter, OD: optical density) and (b) a photo of the setup. (c) Calculated reflectance versus incident angle in the N-BK7 prism based Kretschmann configuration for SPR excitation at $\lambda_{\mathrm{em}}\left(\varepsilon_{\mathrm{r}}^{\mathrm{Ag}}\right.$ and $\varepsilon_{\mathrm{r}}^{\mathrm{Au}}$ : relative permittivity for $\mathrm{Ag}$ and $\mathrm{Au}[38,39], \Gamma$ : the depth-to-width ratio of the dip). (d) Prism-aided redirection of the SPCE directional rays of light.

Figure $2 \mathrm{c}$ shows the computed optical reflectance versus incident angle of light in the $\mathrm{N}$-BK7 prism-based Kretschmann configuration with the bimetallic film, i.e., $2 \mathrm{~nm}$ thick $\mathrm{Au} / 50 \mathrm{~nm}$ thick Ag layers, assumed for SPR excitation at $\lambda_{\mathrm{em}}$ (not $\left.\lambda_{\text {ex }}\right)$. The incident angle at the reflectance minimum being calculated as $43.7^{\circ}$ is considered the same as the angle of the SPCE light at $\lambda_{\mathrm{em}}$ [40]. The depth-to-width ratio of the reflection dip, i.e., the $\Gamma$ which is in proportion to the plasmonic resonance quality factor, turns out to be comparable to that of the single Ag film with the thickness optimized. This bimetallic film nearly optimizes the efficiency of the dye-metal film coupling into the SPCE while providing the Au surface flexibility for biochemical treatment required for the sandwich immune reaction. Figure $2 \mathrm{~d}$ 
depicts the prism-based redirection of the SPCE induced directional rays of light on a given triangular cross section. The ray-surface angles at which light exits the prism base surface reaches $\sim 90^{\circ}$ (nearly zero incident angles), permitting nearly full transmission through air-prism boundary with negligible internal reflection.

The light collection optics comprises a plano-convex lens of a $30 \mathrm{~mm}$ focal length, the emission filter (EMF) (Chroma Co., Bellows Falls, VT, USA) with its pass band of $525 \pm 25 \mathrm{~nm}$, and a neutral density (ND) filter. The collected light is then detected by a photomultiplier tube (PMT) (PMM02, Thorlabs Inc., Newton, MA, USA) connected with a multi-meter (Tektronix DMM 4050, 6-1/2 Digit Precision Multimeter, Beaverton, OR, USA) which is interfaced with a data acquisition computer. We set the PMT gain voltage as $0.3 \mathrm{~V}$ and the ND filter optical density (OD) as 0.6 , both to avoid signal saturation and to enable noise floor to be detectable as the minimum possible value. We then keep these parameters fixed during measurement for all concentrations of cTnI used.

It should, however, be mentioned that the fact that the SPCE produces the 3-dimensional conical direction of light rays must generate the complex internal reflections, given the asymmetrical inner structure of the prism, such as reflections azimuthally slanted and skew from those shown in Figure 2d. This would incur the far-from-zero incident angles of rays at the prism base-air interface, leading to inevitable loss of light for detection in the present setup.

\section{Results and Discussion}

Figure $3 a-d$ show the net fluorescence signal versus $c T n I$ concentrations, under various optical powers of excitation light incident to the chip, i.e., 2, 5.8, 10, and $17 \mathrm{~mW}$. The net signal (vertical axis) is obtained by subtracting the signal measured without cTnI, namely, the background noise from the one measured with cTnI, eliminating effects of autofluorescence and unwanted leak of excitation light through a spectral filter into a detector. Red solid circles represent the fluorescence signal detected through the prism based SPCE chip, while black solid squares represent the reference fluorescence signal detected in the same manner using the control chip at each cTnI concentration. This allows us to compare the net fluorescence signals obtained between two techniques, i.e., the presented SPCE technology and a conventional fluorescence one.

It is found that use of the control chips hardly distinguish the signals from the different concentrations of cTnI while use of the SPCE chips do. This is primarily due to the photobleaching and the saturation effects of the excited dye molecules on the control chip, which began to be significant already at the low concentrations used.

In contrast, the SPCE chip produces much higher signal-to-noise $(\mathrm{S} / \mathrm{N})$ ratio than the control chip at each concentration of $\mathrm{CTnI}$ for all incident excitation powers. This enables the signals from different concentrations to be markedly distinguishable, especially at the low concentration range as shown in Figure 3a-d. The LOD is defined as the concentration that produces the signal corresponding to three times the standard deviation at zero concentration. It is estimated as $22.2,21.7,21.2$, and $26.5 \mathrm{ag} \mathrm{mL}^{-1}$ for excitation powers of $2,5.8,10$, and $17 \mathrm{~mW}$ with coefficient of variation $(\mathrm{CV})<5.2 \%$, respectively. For LOD estimation, we use linear regression of the data at small concentration close to zero, being considered a good approximation as seen in Figure 3a-d. These levels of LODs presented are recognized as the lowest ever reported to date amid those obtained using optical technologies, such as label-free optical sensors and luminescence $[10,19,22,41-45]$ as shown in Table 1, where all LODs were reported using PBS without human serum. 

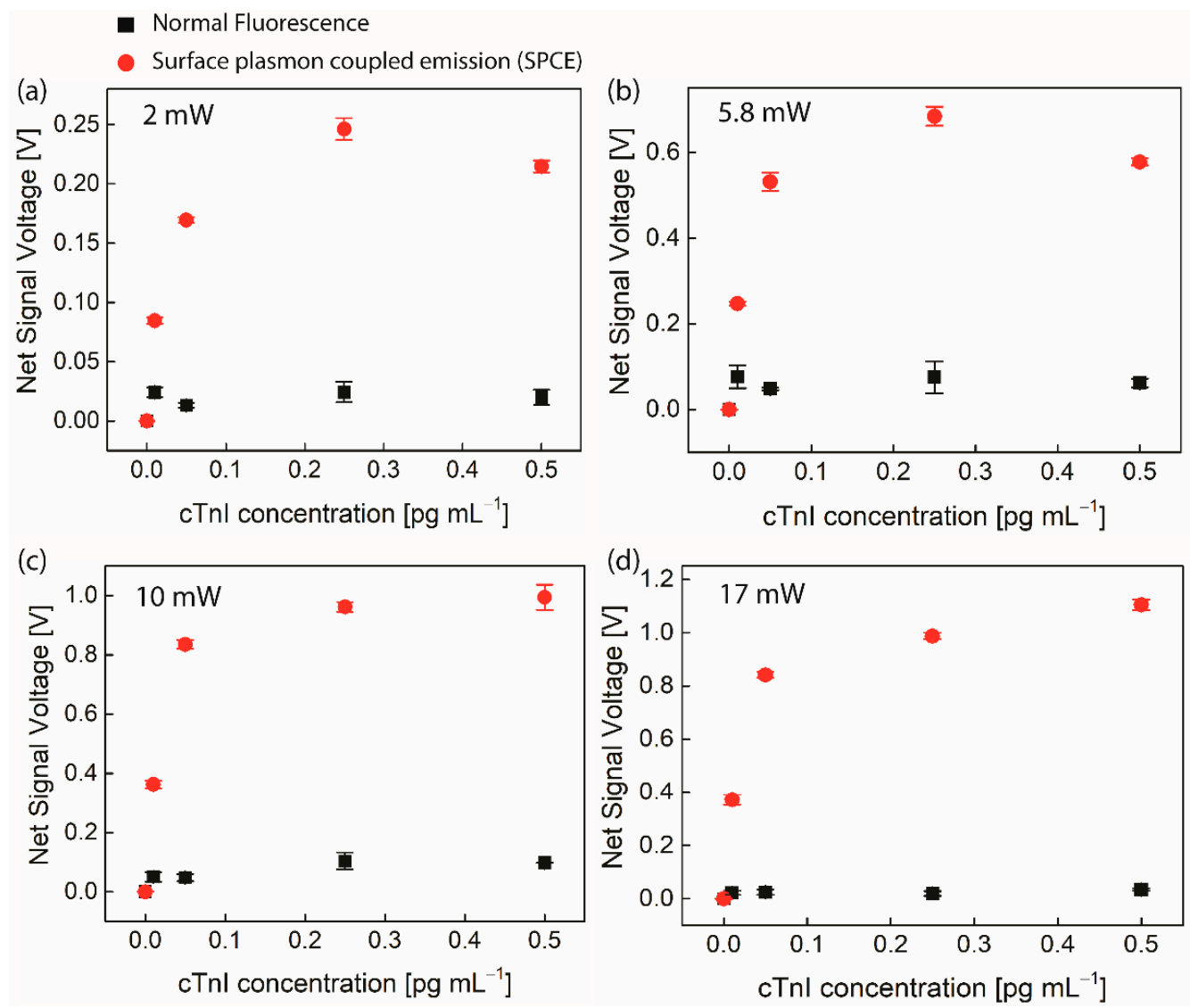

Figure 3. The net fluorescence signal versus $\mathrm{cTnI}$ concentration under various incident powers of excitation source (a) $2 \mathrm{~mW}$, (b) $5.8 \mathrm{~mW}$, (c) $10 \mathrm{~mW}$, and (d) $17 \mathrm{~mW}$. Error bar $= \pm S D$ and $n=3$.

Table 1. The comparison of this work and previous report for cTnI detection using optical technologies.

\begin{tabular}{cccc}
\hline cTnI Detection Methods & Concentration Range & LOD (with No Serum Used) & Ref. \\
\hline Surface plasmon coupled emission (SPCE) & $0-0.5 \mathrm{pg} \mathrm{mL}^{-1}$ & $21.1 \mathrm{ag} \mathrm{mL}^{-1}$ & Present work \\
Optical microfiber coupler & $2-10 \mathrm{fg} \mathrm{mL}^{-1}$ & $2 \mathrm{fg} \mathrm{mL}^{-1}$ & {$[10]$} \\
Chemiluminescence & $0-1.5 \mathrm{ng} \mathrm{mL}^{-1}$ & $0.012 \mathrm{ng} \mathrm{mL}^{-1}$ & {$[19]$} \\
Electrochemiluminescence & $1 \mathrm{fg} \mathrm{mL}-1-1 \mu \mathrm{gL} \mathrm{mL}^{-1}$ & $0.58 \mathrm{fg} \mathrm{mL}^{-1}$ & {$[41]$} \\
Photoelectrochemistry & $0.0005-1000 \mathrm{ng} \mathrm{mL}^{-1}$ & $0.18 \mathrm{pg} \mathrm{mL}^{-1}$ & {$[42]$} \\
Fluorescence & $0.05-32 \mathrm{ng} \mathrm{mL}^{-1}$ & $0.032 \mathrm{ng} \mathrm{mL}^{-1}$ & {$[43]$} \\
Magnetic field-assisted Surface plasmon & $50-125 \mu \mathrm{g} \mathrm{mL}^{-1}$ & $1.25 \mathrm{ng} \mathrm{mL}^{-1}$ & {$[44]$} \\
resonance & $0-0.160 \mu \mathrm{g} \mathrm{mL}^{-1}$ & $0.068 \mathrm{ng} \mathrm{mL}^{-1}$ & {$[22]$} \\
Surface plasmon resonance & $0.005-10 \mathrm{ng} \mathrm{mL}^{-1}$ & $5 \mathrm{and} 10 \mathrm{pg} \mathrm{mL}^{-1}$ & {$[45]$} \\
\hline
\end{tabular}

It is worth noting that the SPCE technologies not only enhance the fluorescent signals detected but also suppress photobleaching due to the near field coupling of excited state dye molecules with thin metal layers that support surface plasmon resonance. To comprehend the superior performance of the SPCE chip over the control chip, let us begin by considering that the LED light source excites the molecules of the dye, the Alexa 488, which are conjugated with the detection antibody (D-Ab@Alexa 488) for the sandwich immune reaction. The excited states of dye molecules make a near-field coupling with the bimetallic film for surface plasmons at $\lambda_{\mathrm{em}}$. The non-radiative coupling of the excited dye molecules into surface plasmons at the dielectric-metal interface, a sort of non-radiative decay of a dye molecule, occurs only for those spaced close to the metal film, i.e., those within the evanescent field depth from the outermost metal $(\mathrm{Au})$ surface of the SPCE chip. This non- 
radiative decay rate $\left(\gamma_{\mathrm{sp}}\right)$ that can be boosted by the enhanced density of local photonic modes of surface plasmons [46,47] can contribute to the total decay rates $\left(\gamma_{t}\right)$ of a dye emitter as given by

$$
\gamma_{\mathrm{t}}=\gamma_{\mathrm{se}}+\gamma_{\mathrm{sp}}+\gamma_{\mathrm{nr}}
$$

where the $\gamma_{\mathrm{se}}$ is the radiative decay rate due to the spontaneous emission while we group the other possible non-radiative decay processes with the single rate constant $\gamma_{\mathrm{nr}}$. Eventually, the presence of non-radiative decay channel, $\gamma_{\mathrm{sp}}$, would produce the directional emission of light at a specific angle into the prism (see Figure 2d) at the same wavelength as the dye fluorescent emission as is termed SPCE. The prism-based light collection would harvest the benefits of the directional emission for light collection efficiency. Therefore, the favorable contribution of the SPCE with its directional properties to the fluorescence detection would increase the $\mathrm{S} / \mathrm{N}$ ratio.

Moreover, this non-radiative decay channel driven by $\gamma_{\mathrm{sp}}$ decreases the probability of coupling between excited dye molecules themselves, leading to photobleaching suppression. As demonstrated in Figure $3 a-d$, the SPCE chip reduces photobleaching significantly while the control chips severely suffer it with increasing the concentration.

It is interesting to note that, though signal suffers from severe saturation, it still rises with concentration increasing to the highest one $\left(0.5 \mathrm{pg} \mathrm{mL}^{-1}\right)$ only for higher excitation powers used. It is not clear for this behavior but the presence of elastic scattering of excitation light with cTnI/D-Ab@Alexa 488, which can in turn leak through the emission filter, may be one of the reasons. This noise cannot be ruled out by the process of subtracting the background noise from the measured signal.

We here define the enhancement factor $(\eta)$ of the detected fluorescence as given by

$$
\eta \equiv \mathrm{S}_{\mathrm{SPCE}} / \mathrm{S}_{\mathrm{control}}
$$

where the $\mathrm{S}_{\mathrm{SPCE}}$ is the net signal (voltage) obtained with the SPCE-chip while the $\mathrm{S}_{\text {control }}$ is that obtained with the control chip at each concentration as seen in Figure 3a-d. The bimetallic SPCE immunoassay technologies permit us to achieve the significant enhancement under each incident excitation power as shown in Figure S1 (Section 1 of Supplementary Materials). We achieved the maximum $\eta$ of $\sim 50$-fold when using the highest incident power that produces the largest number of excited dye molecules that can, in turn, possibly lead to the largest number of the SPCE-generated photons.

The fitting curves to the measured data for quantitative detection of cTnI are also shown in Figure S2. The function used for calibration purpose fitting describes the signal saturation as significantly weakened compared to the control case. The net fluorescence signal still gets higher at higher concentrations, though its rate reduces with increasing cTnI concentration due to the non-vanishing effects of photobleaching.

In these SPCE technologies, excitation photons that do not excite dye molecules are not allowed to directly couple into the prism across the metallic layers due largely to the metal induced light attenuation $(\sim 87 \%)$. This attenuation effect can eliminate the need of using optical component that separates the excitation light from fluorescence signal, such as a dichroic beam splitter commonly found in a typical fluorescence-based lab-on-chip to suppress the noise power at $\lambda_{\mathrm{ex}}$. The quasi-straight geometry of optical components aligned for detecting SPCE (with no beam splitter) from an excitation light source to a detector (see Figure 2a)) would facilitate packaging into the lab-on-chip setup of a simpler form than the typical one.

It is also worth noting that the SPCE quenching would occur for the dye molecules spaced less than $10 \mathrm{~nm}$ from the metal surface due to the non-radiative energy transfer to the metal similar to the Förster resonance energy transfer (FRET) [29]. The layers of the cTnI capture antibody $(\mathrm{C}-\mathrm{Ab})$ and the analyte $(\mathrm{cTnI})$, however, would act as the spacer layers to reduce such quenching effects in this SPCE fluorescence based on sandwich immune reaction. 
Meanwhile, the fact that the excited dye molecules cannot produce the s-polarized SPCE limits the further enhancement of $\mathrm{S} / \mathrm{N}$ ratio in fluorescence detection. The waveguide layer containing the dye-molecules, instead, might be added on the bimetallic film for the s-polarized SPCE coupling into the direction emission [48], though it would not be straightforward to incorporate the dye and the protein elements comprising the sandwich immune reaction into the waveguide.

The time taken for procedures of sandwich reaction after having C-Ab immobilized on the surface, e.g., the bond between $\mathrm{C}-\mathrm{Ab}$ and $\mathrm{cTnI}(\sim 30 \mathrm{~min})$, and that between $\mathrm{cTnI}$ and D-Ab@Alexa 488 ( $\sim 60 \mathrm{~min})$ is $\sim 90 \mathrm{~min}$. This leads to the possibility that, given the dynamic antibodies developed to shorten the immune reaction time, the ultrahigh sensitivity available by the presented SPCE technologies would make it plausible to diagnose the cTnI level in blood in amuch earlier stage.

It is also worthwhile to note that the LOD, however, may be likely to be worse than the current one when using human sera that contain various kinds of proteins. This is because they can induce unwanted non-specific binding (false signal) that would increase the background signal noise. Use of surface rinsing and blocking medium included in the surface treatment of the present work may reduce such unwanted noise and thus prevent the very high sensitivity demonstrated in the present device from being tarnished.

\section{Conclusions}

This work demonstrates the SPCE based fluorescence detection for quantitative assay of cTnI using the sandwich immune reaction. With greatly suppressed photo-bleaching, remarkably enhanced fluorescence is detected with the enhancement factor of up to 50fold. The dye-bimetallic film near-field coupling produces the SPCE induced directional emission at $\lambda_{\mathrm{em}}$, collected by a prism without a dichroic beam splitter commonly used in a typical lab-on-chip fluorescence device. Signal fluctuations of zero concentration turns out to be much smaller than the differential change that occurs as concentration rises to the minimum concentration used. This leads us to estimate the detection limit of $21.2 \mathrm{ag} \mathrm{mL}^{-1}$ being the lowest ever reported to date amid those obtained by optical technologies, such as label-free optical bio-sensing and luminescence-based assay where cTnI in PBS buffer is used (not in human sera).

It is also noted that the fact that the near field coupling cannot occur for the s-polarized SPCE limits further enhancement in detecting fluorescence. However, further enhancement may be accomplished by adding a waveguide layer on top of the bimetallic film to simultaneously obtain the s-polarized directional emission in addition to the p-polarized one for even higher sensitivity [27,48-50]. In this case, dipoles of the fluorescent dyes oriented in parallel to the s-polarization can also be coupled with waveguide modes, subsequently producing s-polarized direction emission at an angle different from the angle of SPCE directional emission. This may additionally benefit early diagnosis of $\mathrm{cTnI}$ to shorten the whole sandwich immune reaction via using dynamic antibodies that can bond with $\mathrm{cTnI}$ within a much shorter reaction time while approximately maintaining the bio-affinity and bond strength.

This work presented does not use human sera that would contain many other kinds of proteins, while focusing on adaptability of the SPCE technologies to cTnI sandwich assay using pure $\mathrm{cTnI}$ in PBS buffer. The protocol of the sensing surface treatment that includes the sandwich immunoassay with DI water rinsing and use of blocking solution, however, would be able to detect cTnI specifically together with minimizing non-specific adsorption under presence of other substances such as in human sera. This can strengthen utility of the ultrahigh sensitivity of the sensor transduction demonstrated in the present work.

Future works shall cover the clinical application of the presented SPCE technologies for cTnI detection in human sera, expecting the LOD to be about an order-of-magnitude higher than the atto-g mL $\mathrm{mL}^{-1}$ level at worst. However, this level still would be very low (still good sensitivity), deserving further investigation with the surface treatment to remove 
unwanted non-specific bonding. In addition, use of dynamic antibodies may shorten the detection time considerably, being more favorable for early diagnosis of cTnI.

The technologies presented for the ultrahigh sensitivity of cTnI assay can also potentially open up an opportunity of removing the need that patients with emergent cardiovascular diseases, such as acute coronary syndromes, should wait several hours until their level in blood rises to the level detectable by the assay devices and kits currently available in the emergency medical center.

Supplementary Materials: The following are available online at https:/ /www.mdpi.com/article/10 .3390/biomedicines9050448/s1, Figure S1: The enhancement factor ( $\eta$ ) of the detected fluorescence, Figure S2: The curve fitting function of measured data for quantitative detection of cTnI.

Author Contributions: V.T.T.: Methodology, Investigation, Data acquisition, Initial analysis, Writing original draft. H.J.: Conceptualization, Supervision, Funding acquisition, Resources, Final analysis, Correcting and finalizing the manuscript. All authors have read and agreed to the published version of the manuscript.

Funding: This work was funded by the National Research Foundation of Korea (NRF) grant funded by the Korean government (MSIT) (grant number: 2020R1F1A1050885), and also supported by the Gachon University research fund of 2019 (grant number: GCU-2019-0803).

Institutional Review Board Statement: Not applicable.

Informed Consent Statement: Not applicable.

Data Availability Statement: The data presented in this study are available on request from the corresponding author. The data are not publicly available due to privacy.

Conflicts of Interest: The authors declare report no declaration of interest.

\section{References}

1. Kottwitz, J.; Bruno, K.A.; Berg, J.; Salomon, G.R.; Fairweather, D.; Elhassan, M.; Baltensperger, N.; Kissel, C.K.; Lovrinovic, M.; Baltensweiler, A.; et al. Myoglobin for Detection of High-Risk Patients with Acute Myocarditis. J. Cardiovasc. Transl. 2020, 13, 853-863. [CrossRef] [PubMed]

2. Matveeva, E.; Gryczynski, Z.; Gryczynski, I.; Malicka, J.; Lakowicz, J.R. Myoglobin Immunoassay Utilizing Directional Surface Plasmon-Coupled Emission. Anal. Chem. 2004, 76, 6287-6292. [CrossRef] [PubMed]

3. Kumar, V.; Brent, J.R.; Shorie, M.; Kaur, H.; Chadha, G.; Thomas, A.G.; Lewis, E.A.; Rooney, A.P.; Nguyen, L.; Zhong, X.L.; et al. Nanostructured Aptamer-Functionalized Black Phosphorus Sensing Platform for Label-Free Detection of Myoglobin, a Cardiovascular Disease Biomarker. ACS Appl. Mater. Interfaces 2016, 8, 22860-22868. [CrossRef] [PubMed]

4. Lee, J.S.; Joung, H.-A.; Kim, M.-G.; Park, C.B. Graphene-Based Chemiluminescence Resonance Energy Transfer for Homogeneous Immunoassay. ACS Nano 2012, 6, 2978-2983. [CrossRef]

5. Yeh, E.T. High-sensitivity C-reactive protein as a risk assessment tool for cardiovascular disease. Clin. Cardiol. 2006, 28, 408-412. [CrossRef]

6. Grabowska, I.; Sharma, N.; Vasilescu, A.; Iancu, M.; Badea, G.; Boukherroub, R.; Ogale, S.; Szunerits, S. Electrochemical Aptamer-Based Biosensors for the Detection of Cardiac Biomarkers. ACS Omega 2018, 3, 12010-12018. [CrossRef]

7. Zhang, H.; Han, Z.; Wang, X.; Li, F.; Cui, H.; Yang, D.; Bian, Z. Sensitive Immunosensor for N- Terminal Pro-brain Natriuretic Peptide Based on N-(Aminobutyl)-N-(ethylisoluminol)-Functionalized Gold Nanodots/Multiwalled Carbon Nanotube Electrochemiluminescence Nanointerface. Analyst 2015, 7, 7599-7604. [CrossRef]

8. Takashio, S.; Yamamuro, M.; Izumiya, Y.; Hirakawa, K.; Marume, K.; Yamamoto, M.; Ueda, M.; Yamashita, T.; Ishibashi-Ueda, H.; Yasuda, S.; et al. Diagnostic utility of cardiac troponin T level in patients with cardiac amyloidosis. ESC Heart Fail. 2018, 5, 27-35. [CrossRef]

9. Song, K.-S.; Nimse, S.B.; Sonawane, M.D.; Lin, Y.; Zhou, Z.; Kim, T. A glass fibre membrane platform for ultra-sensitive detection of cardiac troponin T. Analyst 2017, 142, 3816-3821. [CrossRef]

10. Zhou, W.; Li, K.; Wei, Y.; Hao, P.; Chi, M.; Liu, Y.; Wu, Y. Ultrasensitive label-free optical microfiber coupler biosensor for detection of cardiac Troponin I based on interference turning point effect. Biosens. Bioelectron. 2018, 106, 99-104. [CrossRef]

11. Spain, E.; Carrara, S.; Adamson, K.; Ma, H.; Kennedy, R.O.; Cola, L.D.; Forster, R.J. Cardiac Troponin I: Ultrasensitive Detection Using Faradaic Electrochemical Impedance. ACS Omega 2018, 3, 17116-17124. [CrossRef]

12. Fathil, M.F.M.; Arshad, M.K.M.; Gopinath, S.C.B.; Hashim, U.; Adzhri, R.; Ayub, R.M.; Ruslinda, A.R.; Nuzaihan, M.N.M.; Azman, A.H.; Zaki, M.; et al. Diagnostics on acute myocardial infarction: Cardiac troponin biomarkers. Biosens. Bioelectron. 2015, 70, 209-220. [CrossRef] 
13. Sabek, J.; Martínez-Pérez, P.; García-Rupérez, J. Computational binding study of cardiac Troponin I antibody towards cardiac versus skeletal Troponin I. Comput. Biol. Chem. 2019, 80, 147-151. [CrossRef]

14. Nandhikonda, P.; Heagy, M.D. An Abiotic Fluorescent Probe for Cardiac Troponin I. J. Am. Chem. Soc. 2011, 133, 14972-14974. [CrossRef]

15. Lv, H.; Li, Y.; Zhang, X.; Li, X.; Xu, Z.; Chen, L.; Li, D.; Dong, Y. Thionin functionalized signal amplification label derived dual-mode electrochemical immunoassay for sensitive detection of cardiac Troponin I. Biosens. Bioelectron. 2019, 133, 72-78. [CrossRef]

16. Sun, D.; Luo, Z.; Lu, J.; Zhang, S.; Che, T.; Chen, Z.; Zhang, L. Electrochemical dual-aptamer-based biosensor for nonenzymatic detection of cardiac Troponin I by nanohybrid electrocatalysts labeling combined with DNA nanotetrahedron structure. Biosens. Bioelectron. 2019, 134, 49-56. [CrossRef]

17. Fathil, M.F.M.; Arshad, M.K.M.; Ruslinda, A.R.; Gopinath, S.C.B.; Nuzaihan, M.N.M.; Adzhri, R.; Hashim, U.; Lam, H.Y. Substrate-gate coupling in ZnO-FET biosensor for cardiac Troponin I detection. Sens. Actuators B Chem. 2017, 242, 1142-1154. [CrossRef]

18. Sarangadharan, I.; Regmi, A.; Chen, Y.-W.; Hsu, C.-P.; Chen, P.-C.; Chang, W.; Lee, G.-Y.; Chyi, J.-I.; Shiesh, S.-C.; Lee, G.-B.; et al. High sensitivity cardiac Troponin I detection in physiological environment using AlGaN/GaN High Electron Mobility Transistor (HEMT) Biosensors. Biosens. Bioelectron. 2018, 100, 282-289. [CrossRef]

19. Gopinathan, P.; Sinha, A.; Chung, Y.-D.; Shiesh, S.-C.; Lee, G.-B. Optimization of an enzyme linked DNA aptamer assay for cardiac Troponin I detection: Synchronous multiple sample analysis on an integrated microfluidic platform. Analyst 2019, 144, 4943-4951. [CrossRef]

20. Kar, P.; Pandey, A.; Greer, J.J.; Shankar, K. Ultrahigh sensitivity assays for human cardiac Troponin $\mathrm{I}_{\text {using }} \mathrm{TiO}_{2}$ nanotube arrays. Lab Chip 2012, 12, 821-828. [CrossRef]

21. Miao, L.; Jiao, L.; Tang, Q.; Li, H.; Zhang, L.; Wei, Q. A nanozyme-linked immunosorbent assay for dual-modal colorimetric and ratiometric fluorescent detection of cardiac Troponin I. Sens. Actuators B Chem. 2019, 288, 60-64. [CrossRef]

22. Kwon, Y.-C.; Kim, M.-G.; Kim, E.-M.; Shin, Y.-B.; Lee, S.-K.; Lee, S.D.; Cho, M.-J.; Ro, H.-S. Development of a surface plasmon resonance-based immunosensor for the rapid detection of cardiac Troponin I. Biotechnol. Lett. 2011, 33, 921-927. [CrossRef] [PubMed]

23. Çimen, D.; Bereli, N.; Günaydın, S.; Denizli, A. Detection of cardiac troponin-I by optic biosensors with immobilized anticardiac troponin-I monoclonal antibody. Talanta 2020, 219, 121259. [CrossRef] [PubMed]

24. Lee, W.; Jung, J.; Hahn, Y.K.; Kim, S.K.; Lee, Y.; Lee, J.; Lee, T.-H.; Park, J.-Y.; Seo, H.; Lee, J.N.; et al. A centrifugally actuated point-of-care testing system for the surface acoustic wave immunosensing of cardiac Troponin I. Analyst 2013, 138, $2558-2566$. [CrossRef] [PubMed]

25. Mulpur, P.; Yadavilli, S.; Rao, A.M.; Kamisetti, V.; Podila, R. $\mathrm{MoS}_{2} / \mathrm{WS}_{2} / \mathrm{BN}-$ Silver Thin-Film Hybrid Architectures Displaying Enhanced Fluorescence via Surface Plasmon Coupled Emission for Sensing Applications. ACS Sens. 2016, 1, 826-833. [CrossRef]

26. Tran, N.H.T.; Trinh, K.T.L.; Lee, J.-H.; Yoon, W.J.; Ju, H. Reproducible Enhancement of Fluorescence by Bimetal Mediated Surface Plasmon Coupled Emission for Highly Sensitive Quantitative Diagnosis of Double-Stranded DNA. Small 2018, 1801385. [CrossRef]

27. Calander, N. Theory and Simulation of Surface Plasmon-Coupled Directional Emission from Fluorophores at Planar Structures. Anal. Chem. 2004, 76, 2168-2173. [CrossRef]

28. Enderlein, J.; Ruckstuhl, T. The efficiency of surface-plasmon coupled emission for sensitive fluorescence detection. Opt. Express 2005, 13, 8855-8865. [CrossRef]

29. Trnavsky, M.; Enderlein, J.; Ruckstuhl, T.; McDonagh, C.; MacCraith, B.D. Experimental and theoretical evaluation of surface plasmon-coupled emission for sensitive fluorescence detection. J. Biomed. Opt. 2008, 13, 054021. [CrossRef]

30. Rangełowa-Jankowska, S.; Jankowski, D.; Bogdanowicz, R.; Grobelna, B.; Bojarski, P. Surface Plasmon-Coupled Emission of Rhodamine 110 Aggregates in a Silica Nanolayer. J. Phys. Chem. Lett. 2012, 3, 3626-3631. [CrossRef]

31. Nu, T.T.V.; Tran, N.H.T.; Nam, E.; Nguyen, T.T.; Yoon, W.J.; Cho, S.; Kim, J.; Chang, K.-A.; Ju, H. Blood-based immunoassay of tau proteins for early diagnosis of Alzheimer's disease using surface plasmon resonance fiber sensors. RSC Adv. 2018, 8, 7855-7862. [CrossRef]

32. Tran, V.T.; Yoon, W.J.; Lee, J.-H.; Ju, H. DNA sequence-induced modulation of bimetallic surface plasmons in optical fibers for sub-ppq (parts-per-quadrillion) detection of mercury ions in water. J. Mater. Chem. A 2018, 6, 23894-23902. [CrossRef]

33. Kim, J.; Kim, S.; Nguyen, T.T.; Lee, R.; Li, T.; Yun, C.; Ham, Y.; An, S.S.A.; Ju, H. Label-Free Quantitative Immunoassay of Fibrinogen in Alzheimer Disease Patient Plasma Using Fiber Optical Surface Plasmon Resonance. J. Electron. Mater. 2016, 45, 2354-2360. [CrossRef]

34. Nguyen, T.T.; Bea, S.O.; Kim, D.M.; Yoon, W.J.; Park, J.-W.; An, S.S.A.; Ju, H. A regenerative label-free fiber optic sensor using surface plasmon resonance for clinical diagnosis of fibrinogen. Int. J. Nanomed. 2015, 10, 155-163.

35. Ong, B.H.; Yuan, X.; Tan, Y.Y.; Irawan, R.; Fang, X.; Zhang, L.; Tjin, S.C. Two-layered metallic film-induced surface plasmon polariton for fluorescence emission enhancement in on-chip waveguide. Lab Chip 2007, 7, 506-512. [CrossRef]

36. Mabe, T.; Zeng, Z.; Bagra, B.; Ryan, J.; Wei, J. Surface Plasmon Resonance of A Bimetallic Nanostructured Film for Enhanced Optical Sensitivity. ChemistrySelect 2018, 3, 3018-3023. [CrossRef] 
37. Wang, L.; Gaigalas, A.K.; Reipa, V. Optical properties of Alexa ${ }^{\mathrm{TM}} 488$ and Cy ${ }^{\mathrm{TM}} 5$ immobilized on a glass surface. Biotechniques 2005, 38, 127-132. [CrossRef]

38. McPeak, K.M.; Jayanti, S.V.; Kress, S.J.P.; Meyer, S.; Iotti, S.; Rossinelli, A.; Norris, D.J. Plasmonic Films Can Easily Be Better: Rules and Recipes. ACS Photonics 2015, 2, 326-333. [CrossRef]

39. Ciesielski, A.; Skowronski, L.; Trzcinski, M.; Górecka, E.; Trautman, P.; Szoplik, T. Evidence of germanium segregation in gold thin films. Surf. Sci. 2018, 674, 73-78. [CrossRef]

40. Weber, W.H.; Eagen, C.F. Energy transfer from an excited dye molecule to the surface plasmons of an adjacent metal. Opt. Lett. 1979, 4, 236-238. [CrossRef]

41. Wang, S.; Zhao, Y.; Wang, M.; Li, H.; Saqib, M.; Ge, C.; Zhang, X.; Jin, Y. Enhancing Luminol Electrochemiluminescence by Combined Use of Cobalt-Based Metal Organic Frameworks and Silver Nanoparticles and Its Application in Ultrasensitive Detection of Cardiac Troponin I. Anal. Chem. 2019, 91, 3048-3054. [CrossRef] [PubMed]

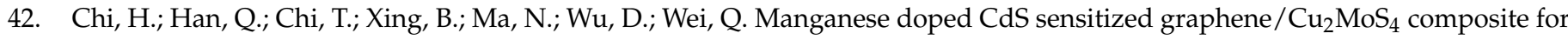
the photoelectrochemical immunoassay of cardiac Troponin I. Biosens. Bioelectron. 2019, 132, 1-7. [CrossRef] [PubMed]

43. Lou, D.; Fan, L.; Cui, Y.; Zhu, Y.; Gu, N.; Zhang, Y. Fluorescent Nanoprobes with Oriented Modified Antibodies to Improve Lateral Flow Immunoassay of Cardiac Troponin I. Anal. Chem. 2018, 90, 6502-6508. [CrossRef] [PubMed]

44. Wu, Q.; Sun, Y.; Zhang, D.; Li, S.; Zhang, Y.; Ma, P.; Yu, Y.; Wang, X.; Song, D. Ultrasensitive magnetic field-assisted surface plasmon resonance immunoassay for human cardiac Troponin I. Biosens. Bioelectron. 2017, 96, 288-293. [CrossRef]

45. Diware, M.S.; Cho, H.M.; Chegal, W.; Cho, Y.J.; Kim, D.S.; Kim, K.S.; Paek, S.H. Ultrasensitive, label-free detection of cardiac biomarkers with optical SIS sensor. Biosens. Bioelectron. 2017, 87, 242-248. [CrossRef]

46. Baumberg, J.J.; Aizpurua, J.; Mikkelsen, M.H.; Smith, D.R. Extreme nanophotonics from ultrathin metallic gaps. Nat. Mater. 2019, 18, 668-678. [CrossRef]

47. Muskens, O.L.; Giannini, V.; Sánchez-Gil, J.A.; Rivas, J.G. Strong Enhancement of the Radiative Decay Rate of Emitters by Single Plasmonic Nanoantennas. Nano Lett. 2007, 7, 2871-2875. [CrossRef]

48. Gryczynski, I.; Malicka, J.; Nowaczyk, K.; Gryczynski, Z.; Lakowicz, J.R. Effects of Sample Thickness on the Optical Properties of Surface Plasmon-Coupled Emission. J. Phys. Chem. B 2004, 108, 12073-12083. [CrossRef]

49. Badugu, R.; Szmacinski, H.; Ray, K.; Descrovi, E.; Ricciardi, S.; Zhang, D.; Chen, J.; Huo, Y.; Lakowicz, J.R. Metal-Dielectric Waveguides for High-Efficiency Coupled Emission. ACS Photonics 2015, 2, 810-815. [CrossRef]

50. Gryczynski, I.; Malicka, J.; Nowaczyk, K.; Gryczynski, Z.; Lakowicz, J.R. Waveguide-Modulated Surface Plasmon-Coupled Emission of Nile Blue in Poly(Vinyl Alcohol) Thin Films. Thin Solid Films 2006, 510, 15-20. [CrossRef] 